\title{
UTILIZANDO A CONTA DE ENERGIA ELÉTRICA PARA O ENSINO DO PROCESSO DE MULTIPLICAÇÃO ENTRE MATRIZES
}

\section{USING THE ELECTRIC BILL FOR THE TEACHING OF THE MATRIX MULTIPLICATION PROCESS}

\author{
Antonio Ramon Firmo da Costa ${ }^{1}$ \\ Universidade Estadual Vale do Acaraú - UVA \\ Vaniele Sousa Alves ${ }^{2}$ \\ Universidade Estadual Vale do Acaraú - UVA \\ Nilton José Neves Cordeiro ${ }^{3}$ \\ Universidade Estadual Vale do Acaraú - UVA
}

\begin{abstract}
Resumo
Este trabalho relata uma experiência dos autores, um professor e bolsistas do Programa Institucional de Bolsas de Iniciação à Docência (PIBID), da Universidade Estadual Vale do Acaraú (UVA) sobre a aplicação de uma oficina no $1^{\circ}$ ano do ensino médio da escola Doutor João Ribeiro Ramos, localizada na cidade de Sobral, Ceará. A oficina teve como meta o ensino de multiplicação de matrizes utilizando dados da conta de energia elétrica, tendo em vista a possibilidade de mostrar aos alunos que a matemática está presente em situações do dia a dia. A aplicação da oficina se deu em dois encontros. $\mathrm{O}$ primeiro encontro foi reservado para a introdução do conteúdo com breves definições e uma posterior aplicação de um Trabalho Direcionado (TD). O segundo encontro foi iniciado com a aplicação de outro TD para ver se os alunos conseguiam respondê-lo sem qualquer formalização. Ao final fora aplicado um terceiro TD para verificar o que eles aprenderam nos dois encontros. A oficina buscou também usar os erros dos alunos como um elemento desencadeador de questionamento do ensino, ou ainda, como um impulso para aprendizagem. Como resultados verificou-se que a maioria dos alunos compreendeu o que foi repassado, apesar de que muitos ainda apresentaram certas dificuldades nas quatro operações elementares, o que é preocupante, pois é assunto considerado fácil para o nível escolar em que eles se encontravam. Com isso, verificouse que ainda há muito que melhorar no ensino e aprendizagem de matemática e que práticas de ensino alternativas podem ser positivas. Intervenções como esta, viabilizadas por um programa como o PIBID, podem propiciar enriquecimento na formação acadêmica de quem as conduzem, agregando experiência e trazendo mais conhecimentos a partir das dificuldades enfrentadas dentro de uma sala de aula, buscando formas de ajudar os alunos a superarem obstáculos.
\end{abstract}

Palavras-chave: PIBID; Conta de energia; Multiplicação entre matrizes.

\section{Abstract}

This work addresses an experience of the authors, a professor and scholars of the Institutional Scholarship Program (PIBID) of the State University of Vale do Acaraú

\footnotetext{
${ }^{1}$ Email: ramon.arfc@gmail.com

${ }^{2}$ Email: vaniele011@gmail.com

${ }^{3}$ Email: nilton76@gmail.com
} 
(UVA) about an application of a workshop in the 1st. year of high school of DoutorJoão Ribeiro Ramos School, located In the city of Sobral, Ceará. The workshop aims to teach multiplication of matrices in electric energy bill data, in order to show students that mathematics is present in day dialogues. The workshop took place in two meetings. The first meeting was reserved for an introduction of content with brief definitions and a later application of a Directed Work - TD. The second meeting was started with an application of another TD to get the results obtained answer it without any formalization. At the end for a given year TD to check what is learned in the two encounters. The workshop also seeks to use students' mistakes as a triggering factor for questioning teaching, or as an impetus for learning. As a result, it was verified that the majority of the students understood what was taught, although many still presented certain difficulties concerning the four basic operations, which is worrying, since it is considered an easy subject for the school level in which they were. With this, it has been found that there is still much to improve in the mathematics teaching and learning process and that alternative teaching practices can be positive. Interventions such as this one can provide enrichment in the academic formation of those who lead them, adding experience and bringing more knowledge from the difficulties faced in a classroom, seeking ways to help students overcome obstacles.

Keywords: PIBID; Electric bill; Matrix multiplication.

\section{Introdução}

Ainda nos dias de hoje existe um grande desafio no processo de ensino e aprendizagem de matemática no Ensino Básico. Uma maneira de exemplificar isso seria através de um resultado recente e bem negativo para o nosso país: o desempenho em matemática de alunos de 15 e 16 anos obtido pelo Brasil na maior avaliação educacional do mundo, o PISA - Programme for International Student Assessment, regrediu, continuando num patamar baixo desde o início da implantação do programa.

Focando-se no ensino médio, a nossa deficiência em matemática pode ser atestada pelo resultado de 2015, apontado pelo Índice de Desenvolvimento da Educação Básica (IDEB): apenas 7,3\% dos jovens que terminam o $3^{\circ}$ ano têm o conhecimento considerado adequado.

Dentro do ensino e aprendizagem de matemática são grandes as dificuldades encontradas em diversos níveis de escolaridade e nos mais variados conteúdos. Essas adversidades estão presentes, também, no estudo de matrizes, mais especificamente quando se está trabalhando multiplicação entre matrizes.

Há trabalhos como os de Souza, Lopes e Azevedo (2013) e Ávila (2013) que versam sobre o assunto. O primeiro autor cita Cruz (2012), sinalizando que a falta de motivação dos alunos na sala de aula no estudo de matrizes se dá pelo fato de ser explorado apenas cálculos, sem fazer relação entre teoria e prática. Enquanto o segundo 
Antonio Ramon Firmo da Costa, Vaniele Sousa Alves e Nilton José Neves Cordeiro

Utilizando a conta de energia elétrica para o ensino do processo de multiplicação entre matrizes sugere que alunos que dominam as quatro operações básicas - adição, subtração, multiplicação e divisão - aprendem melhor o conteúdo de matrizes.

Diante da existência de problemas como os citados anteriormente, os autores deste trabalho, um professor orientador e bolsistas do Programa Institucional de Bolsas de Iniciação à Docência (PIBID), da Universidade Estadual Vale do Acaraú (UVA), estes até então atuantes, sobre a forma de monitores e aplicadores de oficinas, projetos e observações, na escola de ensino médio Doutor João Ribeiro Ramos, localizada em Sobral, Ceará, vêm, por meio deste trabalhado, relatar uma experiência obtida durante a aplicação de uma oficina intitulada, "O ensino de multiplicação entre matrizes através da conta de energia elétrica", em uma turma do $1^{\circ}$ ano do ensino médio da instituição de ensino citada acima.

Neste sentido, este trabalho tem como objetivo relatar a experiência vivida com aplicação da oficina, destacando pontos positivos, mas também outros preocupantes que foram observados e que devem ser considerados em reflexões acerca do ensino de matemática.

\title{
Caminho Metodológico
}

De uma maneira geral o estudo de matrizes é realizado no $2^{\circ}$ ou $3^{\circ}$ anos do ensino médio. Contudo, concordamos que

\begin{abstract}
Conceitos e habilidades desenvolvem-se no decorrer de longos períodos de tempo. Isso é verdade não apenas para as características gerais do pensamento, tais como foram estudadas por Piaget e outros psicogeneticistas, mas também para os conteúdos das ciências, em particular da Matemática. A lentidão do desenvolvimento do conceito tem sido subestimada pelos professores e pelos currículos. Em geral, aceita-se que, uma vez que os alunos tenham estudado um capítulo da Matemática, devem sabê-lo e, portanto, não seria necessário retomá-lo nos anos escolares seguintes. Entretanto, estudos empíricos tem mostrado que seria mais eficaz estudar a mesma matéria, ano após ano, aprofundando-se cada vez mais, desvelando novos aspectos e retomando aspectos estudados anteriormente. (CARVALHO, 1994, p. 88).
\end{abstract}

Como Carvalho (1994) afirma, habilidades são desenvolvidas ao longo do tempo, e, sendo assim, o estudo de determinado conteúdo pode ser feito de maneira contínua e repetida. Esta atitude pode receber certa resistência por parte de alguns professores que acreditam que o tempo em sala de aula é muito curto para ainda ter este tipo de atitude. 
Antonio Ramon Firmo da Costa, Vaniele Sousa Alves e Nilton José Neves Cordeiro

Utilizando a conta de energia elétrica para o ensino do processo de multiplicação entre matrizes

Dessa forma, parece fazer sentido o projeto "O ensino de multiplicação de matrizes através da conta de energia elétrica" ser aplicado em uma turma de $1^{\circ}$ ano do ensino médio da escola Doutor João Ribeiro Ramos, mesmo crendo que seria um desafio considerável.

A aplicação foi realizada em dois encontros, cada um deles composto de duas aulas de 50 (cinquenta) minutos. O primeiro encontro aconteceu no dia 15 de setembro de 2016, na sala de aula do $1^{\circ}$ ano do ensino médio, turno manhã, turma "A", com 42 alunos dispostos em seis filas de sete alunos cada. De início, foram apresentados o conceito de matriz e alguns tipos especiais de matrizes, tais como, matriz quadrada, triangular, diagonal, entre outras. Além disso, mostrou-se como identificar a igualdade entre matrizes, como se opera a adição e a subtração de matrizes.

Tais definições foram apresentadas por meio de uma intervenção com todos os alunos na sala do $1^{\underline{0}}$ ano "A" da escola em questão, com o apoio da professora responsável pela sala no momento da aplicação. Esse primeiro encontro foi marcado pela forte participação dos alunos. Notou-se, o envolvimento deles principalmente quando perceberam que o conteúdo, matrizes, poderia ser tranquilamente identificado e relacionado com o dia a dia dos discentes. Um momento importante da participação dos alunos se deu no instante em que, definindo matrizes, foi exibida para eles uma imagem (Figura 1) que expunha que até a própria sala de aula poderia ser entendida como exemplo de matriz.

Figura 1 - Exemplo de sala de aula em estrutura matricial

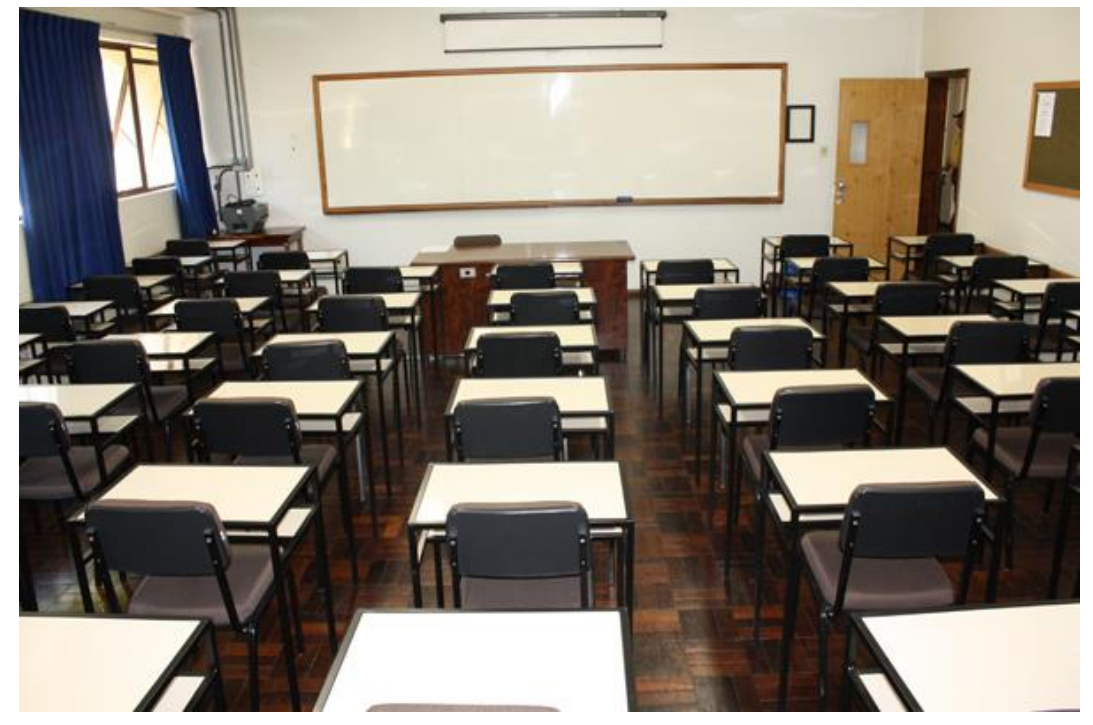

Fonte: UTFPR (2016) 
Antonio Ramon Firmo da Costa, Vaniele Sousa Alves e Nilton José Neves Cordeiro

Utilizando a conta de energia elétrica para o ensino do processo de multiplicação entre matrizes

No momento em que eles viram a imagem, solicitou-se que identificassem

algumas posições de seus colegas que representassem na ocasião elementos de uma matriz. Por exemplo, sabendo que para apontar um componente em uma matriz é preciso saber primeiramente a linha e depois a coluna, solicitou-se que eles mencionassem o nome da pessoa que se encontrava sentada na cadeira correspondente a coluna 5 e linha 2 da sala. Pelo menos cinco situações desse tipo foram formuladas e prontamente respondidas pelos estudantes.

Logo após, ocorreu a explanação dos tipos de matrizes. Esse momento foi marcado pela curiosidade deles em saberem o motivo pelo qual as matrizes tinham nomes específicos. Em dado momento ocorreram alguns questionamentos do tipo: "Por que matriz quadrada", "Por que matriz diagonal" e "Por que matriz identidade". Dentro das possibilidades todas essas perguntas foram respondidas de maneira que os alunos se sentissem satisfeitos com as respostas. Mais adiante, foi apresentado aos alunos como identificar duas matrizes iguais. Este momento merece destaque pelo alto grau de atenção dispensado pelos alunos tanto durante a explicação do conteúdo quanto no exercício dos exemplos utilizados.

Seguindo a oficina, as operações de adição e subtração de matrizes foram abordadas unicamente por meio de exemplos, ressaltando o quão é importante observar se há igualdade de linhas e colunas entre as matrizes para que tais operações pudessem ser efetuadas.

Realizadas todas essas explanações, para efeito avaliativo e análise do que eles aprenderam no primeiro encontro, foi solicitado que os alunos respondessem a um Trabalho Direcionado - TD (Figura 2) composto por uma questão de três itens que envolviam a teoria apresentada até aquele presente momento. 
Figura 2 - Aplicação de um TD

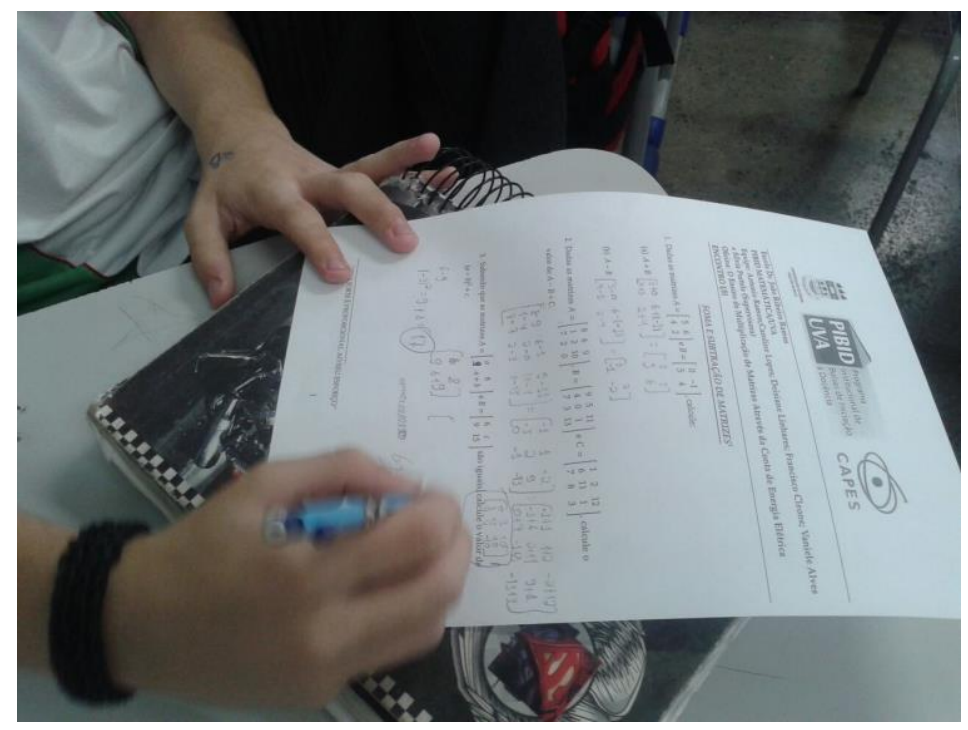

Fonte: Arquivo dos autores

O encontro seguinte aconteceu dia 22 de setembro de 2016, oportunizando aos alunos a ampliação dos conhecimentos adquiridos no primeiro encontro. Desta vez, os estudantes tiveram a chance de aprender como se realiza a multiplicação entre matrizes, que foi o foco da oficina.

Neste segundo momento utilizamos a ideia de que:

[...] a aprendizagem de um novo conceito matemático dar-se-ia pela apresentação de uma situação problema ao aluno, ficando a formalização do conceito como a última etapa do processo de aprendizagem. Nesse caso, caberia ao aluno a construção do conhecimento matemático que permite resolver o problema, tendo o professor como um mediador e orientador do processo ensino-aprendizagem, responsável pela sistematização do novo conhecimento. (BRASIL, 2006, p. 81).

Resolvemos trabalhar com esta estratégia de resolução de problemas, onde podemos reportar Polya (1995), de maneira contextualizada como uma abordagem complementar a utilizada na etapa inicial. Para isso, de início foi solicitado que eles, por meio dos conhecimentos teóricos sobre matrizes já adquiridos, respondessem outro TD com a seguinte situação-problema:

1. No mês de junho Maria teve o consumo de energia de 146kWh em sua casa. Em julho foram $158 \mathrm{kWh}$ e em agosto, $173 \mathrm{kWh}$. João, por sua vez, teve os seguintes 
Antonio Ramon Firmo da Costa, Vaniele Sousa Alves e Nilton José Neves Cordeiro

Utilizando a conta de energia elétrica para o ensino do processo de multiplicação entre matrizes consumos no mesmo período: em junho, $130 \mathrm{kWh}$, em julho, $145 \mathrm{kWh}$ e em agosto, $125 \mathrm{kWh}$.

a) Organize no quadro abaixo os consumos de Maria e João.

\begin{tabular}{|c|c|c|c|}
\hline NOME & JUNHO & JULHO & AGOSTO \\
\hline MARIA & & & \\
\hline JOÃO & & & \\
\hline
\end{tabular}

b) De posse do quadro abaixo que indica o preço do kWh em cada mês, calcule o valor, em $R \$$, do consumo de Maria nos três meses. Logo após, calcule o valor, também em $R \$$, do consumo de João.

\begin{tabular}{|c|c|}
\hline Mês & Preço por $k W h$ \\
\hline Junho & 0,70872 \\
\hline Julho & 0,70078 \\
\hline Agosto & 0,68266 \\
\hline
\end{tabular}

c) Explique com suas palavras como foram realizados os cálculos do item (b).

Como as questões envolviam números decimais e a multiplicação se tornaria um pouco trabalhosa, com o aval da professora, os alunos utilizaram a calculadora do celular. Entre erros e acertos, os alunos levaram pouco mais de dez minutos para finalizarem a tarefa. Depois que os alunos terminaram de responder a situação proposta, solicitou-se que eles entregassem o papel que constava as respostas.

Em seguida foi feito um rápido comentário aos alunos sobre as questões, mas sem que houvesse uma efetiva correção. Entretanto, adiantou-se que o TD aplicado teve a finalidade de fazer com que os alunos compreendessem como se calcula o preço total a ser pago pelo consumo de energia elétrica consumida em um mês, tendo em mãos a quantidade de $\mathrm{kWh}$ consumida no mês e o preço de cada $\mathrm{kWh}$. Vale ressaltar que os valores contidos nos quadros apresentados na situação acima são baseados em situações reais.

Seguindo as atividades do segundo encontro, foi revisado o que havia sido visto no primeiro momento e, em seguida, dado continuidade ao estudo de operações com matrizes. Assim, posteriormente foi realizada a formalização do conceito de 
Antonio Ramon Firmo da Costa, Vaniele Sousa Alves e Nilton José Neves Cordeiro

Utilizando a conta de energia elétrica para o ensino do processo de multiplicação entre matrizes

multiplicação entre matrizes, e, após isso, fora dito abertamente aos alunos que eles já

haviam feito essa operação sem perceber, na situação-problema do consumo de energia.

No que tange a formalização do conceito de multiplicação entre matrizes, primeiro foi mostrado para os alunos as condições para que possa ser feita essa operação. Em seguida, foram feitos alguns exemplos com o intuito de fixar a ideia.

Para finalizar a oficina, foi repassado aos estudantes um terceiro TD contendo questões que envolviam adição, subtração e multiplicação entre matrizes, direcionando para que os mesmos exercitassem ainda mais do conteúdo aprendido, e, além disso, atendesse como um sinalizador do que os alunos assimilaram nos dois encontros da oficina.

\section{Análise dos Trabalhos Direcionados}

A seguir temos uma sucinta análise de como os alunos da turma do $1^{\mathrm{O}}$ ano "A" do ensino médio da escola Doutor João Ribeiro Ramos, se desempenharam para responder os TD's aplicados nos dois encontros. Além disso, realizamos uma averiguação de como os erros dos estudantes poderiam ajudá-los na busca por caminhos corretos até chegaram à solução adequada de uma determinada situação-problema. Ainda seguindo esta linha, vemos que alguns alunos apresentaram certa complicação em relação aos cálculos básicos, dificuldades essas que, segundo Sanchez (2004, apud ALMEIDA, 2006, p.2-3), se manifestam nos aspectos a seguir:

Dificuldades em relação ao desenvolvimento cognitivo e à construção da experiência matemática; do tipo da conquista de noções básicas e princípios numéricos, da conquista da numeração, quanto à prática das operações básicas, quanto à mecânica ou quanto à compreensão do significado das operações. Dificuldades na resolução de problemas, o que implica a compreensão do problema, compreensão e habilidade para analisar o problema e raciocinar matematicamente. [...]

Dificuldades originadas no ensino inadequado ou insuficiente, seja porque a organização do mesmo não está bem sequenciado, ou não se proporcionam elementos de motivação suficientes; seja porque os conteúdos não se ajustam às necessidades e ao nível de desenvolvimento do aluno, ou não estão adequados ao nível de abstração, ou não se treinam as habilidades prévias; seja porque a metodologia é muito pouco motivadora e muito pouco eficaz. (p. 174).

Dificuldades eram esperadas, embora algumas tenham extrapolado as expectativas, levando em consideração que alguns alunos se mostraram alheios em relação a alguns pré-requisitos necessários a aplicação da oficina, como o domínio das 
Antonio Ramon Firmo da Costa, Vaniele Sousa Alves e Nilton José Neves Cordeiro

Utilizando a conta de energia elétrica para o ensino do processo de multiplicação entre matrizes quatro operações básicas. A interpretação de texto também foi um tanto quanto precária, por parte de alguns, como veremos a seguir.

\title{
O primeiro encontro
}

O primeiro encontro foi marcado pela apresentação de algumas definições mais formais no estudo de matrizes, tais como igualdade entre elas e operações com as mesmas. Com isso, foi repassado um TD abordando o que havia sido trabalhado. A maioria dos alunos conseguiu aprender a essência do que havia sido repassado. Isso pode ser verificado pelas respostas dadas às questões que continham no TD.

Entretanto, foram detectados alguns erros que, às vezes, ainda são vistos na análise de respostas de alunos no ensino médio quando o que está em pauta são as operações básicas.

Pinto (1998) afirma que:

\begin{abstract}
Numa avaliação classificatória, em que o foco de atenção está voltado para o acerto da resposta, o erro, por não ser utilizado como um instrumento de reflexão, provavelmente, não será valorizado pelo professor. Em outra concepção de avaliação, preocupada mais com a formação do aluno em termos de aprendizagens significativas e duradouras, o erro deixa de ser apenas uma resposta a ser analisada; passa a ser uma questão desafiadora que o aluno coloca ao professor, portanto, um elemento desencadeador de um amplo questionamento do ensino. Nesse paradigma de avaliação formativa, ao invés de uma avaliação, apenas de resultados, que enfatiza os fracassos e a ausência de aprendizagem pelo aluno, o erro dirige o olhar do professor para o contexto e o processo do conhecimento a ser construído. Avalia-se menos para punir e mais para formar. (p. 10).
\end{abstract}

Seguindo a concepção de avaliação preocupada com a formação do aluno, quando esses erros foram detectados no momento da oficina, buscou-se explorar estes de uma forma que os discentes que estavam errando conseguissem ver o equívoco e como deveriam fazer para seguir outro caminho de resolução. Os erros mais detectados foram a soma e subtração de números naturais. Além disso, foi visto que alguns tinham uma grande dificuldade quando chegavam a uma situação de jogo de sinais.

Dentro das possibilidades e do tempo disponível, buscou-se ajudar os estudantes para que eles conseguissem sanar todas as dúvidas. Ademais, fora chamada a atenção para que eles não repetissem os mesmos erros outrora ocorridos. Essa preocupação com os equívocos deve-se ao fato de que "o erro, tanto como o acerto, é um elemento dinâmico, quando observado pelo aluno tem um papel produtivo no processo de aprendizagem" (PINTO, 1998). Logo, a ideia era fazer com que os discentes se 
Antonio Ramon Firmo da Costa, Vaniele Sousa Alves e Nilton José Neves Cordeiro

Utilizando a conta de energia elétrica para o ensino do processo de multiplicação entre matrizes sentissem seguros para fazerem as questões, e não envergonhados quando acontecesse algo que não estivesse de acordo com os cálculos corretamente feitos.

\section{O segundo encontro}

O segundo encontro iniciou-se com aplicação de um TD onde os alunos deveriam interpretar uma situação-problema e logo após responder três questões. Como pode ser verificado no TD que se encontra na seção Caminho Metodológico, o item "a" é um tanto quanto fácil, pois é pedido que o quadro fosse completado com os dados que aparecem no enunciado da situação-problema. Neste item não houve grandes equívocos. Na realidade, a maioria dos alunos conseguiu realizar esta tarefa com êxito.

O item "b" já é um item com um grau de complexidade maior, pois envolve a multiplicação de números naturais com números decimais. A dificuldade na multiplicação foi minimizada pelo fato de ter sido autorizado o uso da calculadora do celular para realizar as operações. Foi possível observar que alguns estudantes não conseguiram interpretar, de fato, o que o item " $b$ " pedia. Muitos apenas calcularam os valores referentes ao consumo mensal, separadamente. Não obtiveram a resposta final que seria conseguida realizando a adição dos valores obtidos com o produto do número de $\mathrm{kWh}$ consumido no mês pelo respectivo valor que foi apresentado no quadro. Outros deixaram a questão em branco.

O item "c", considerado pelos autores como um dos mais importantes, pois nele constaria a percepção dos alunos quanto ao procedimento nas soluções realizado pelos mesmos, foi o que apresentou uma quantidade bem variada de respostas. Abaixo estão listadas cinco tipos de respostas que nos chamaram atenção e, posteriormente, serão feitos comentários a respeito delas. Destaca-se, antes de tudo, que apenas 25 dos 42 alunos entregaram as folhas com pelo menos dois dos três itens devidamente respondidos.

Resposta 1: "Foram multiplicados os consumos de energia com os preços por kWh. Depois foram todos somados e dado o resultado dos três meses juntos".

Resposta 2: "Usando calculadora".

Resposta 3:"Pegamos o kWh de cada mês e multiplicamos pelo preço do kWh de cada mês, depois somamos o resultado de cada mês com seu preço, aí chegamos no resultado".

Resposta 4: "Houve a multiplicação de dois valores resultando no consumo mensal. Depois houve a soma dos valores dos três meses". 
Resposta 5: "Primeiramente se utiliza o mesmo procedimento de soma para multiplicar em seguida somei o valor para encontrar o resultado".

Analisando a Resposta 1, percebemos que alunos conseguiram pelo menos interpretar a questão e aplicar os conhecimentos que já possuíam em sua vida acadêmica para resolverem o que era pedido no item " $b$ ".

A Resposta 2 demonstra que os alunos que a fizeram não entenderam bem o que o item "c" pedia, mas pelo menos, no que foi observado nas correções dos TD's que constavam essa resposta, foi conseguido realizar o que era pedido no item " $b$ ". O que faltou, pelo que pode ser visto foi apenas uma melhor interpretação do item.

A análise das respostas 3, 4 e 5 são análogas à resposta 1, diferindo apenas na forma como eles colocaram no papel o processo de encontrar a resposta.

Houve um caso em que o aluno não conseguindo interpretar bem o item "c", copiou os cálculos feitos no item "b" e os colou no item "c". Outro tipo de falha cometida no item "c" que se verificou foi quando alguns alunos sugeriram a Resposta 3, atrapalhando-se na interpretação de texto e não entendendo o que o item em questão pedia.

Com relação ao TD que constavam as questões de adição, subtração e multiplicação entre matrizes, foi constatado que a grande maioria dos alunos conseguiu realizar as operações pedidas.

\section{Resultados e Discussões}

Com a aplicação desta oficina percebemos que muitos alunos possuem dificuldades em conteúdos básicos de matemática, tais como as quatro operações elementares. Além disso, foi notório que alguns possuem deficiências de interpretação de texto. Ambas complicações, provavelmente, são advindas de um ensino frágil no nível fundamental.

Entretanto, apesar de todas as dificuldades encontradas nas resoluções dos TD's propostos, ficou evidente que, quando foi trabalhada a aproximação professor-alunoconteúdo, conseguiu-se um desempenho razoavelmente bom. Acreditamos que isso se deve ao fato de que quando os condutores da oficina (que estavam no papel de professores naquele momento) se aproximavam dos alunos e buscavam acompanhar como eles resolveram as questões, os mesmos se sentiam mais à vontade para perguntar e arriscar caminhos para a resolução das questões. 
Antonio Ramon Firmo da Costa, Vaniele Sousa Alves e Nilton José Neves Cordeiro

Utilizando a conta de energia elétrica para o ensino do processo de multiplicação entre matrizes

Ao final, acredita-se que o conteúdo apresentado para os estudantes foi compreendido de maneira que ao chegarem no $2^{\mathrm{o}} \mathrm{e} / \mathrm{ou} 3^{\mathrm{o}}$ anos do ensino médio, momento que serão vistos conteúdos sobre matrizes, potencialmente haverá uma melhor compreensão do estudo de matrizes, visto que, de certa forma, a temática não será novidade para aqueles que se dedicaram e prestaram atenção às explanações e tentaram resolver as questões propostas.

\section{Conclusão}

A aplicação da oficina "O ensino de multiplicação de matrizes através da conta de energia elétrica" oportunizou que fosse verificado que é sim interessante e possível trabalhar de forma "adiantada" conteúdos com alunos do ensino médio.

O presente trabalho relatou a experiência da aplicação de uma oficina que trazia como tema central o ensino de multiplicação entre matrizes no $1^{\circ}$ ano do ensino médio. Tal aplicação demonstrou que, quando a matemática é trabalhada atrelada a conteúdos do dia a dia dos discentes, fica mais fácil para eles entenderem, tendo em vista que quanto mais perto da realidade do aluno estiver o conteúdo, potencialmente será mais fácil aprender. No caso aqui relatado, foi estudado um conteúdo matemático combinado com dados da fatura de energia elétrica.

A oficina também permitiu que fosse verificado que muitos dos educandos ainda possuem dificuldades que não deveriam existir no nível em que eles se encontram. Os obstáculos aqui referidos são as quatro operações e a interpretação de itens simples das questões aplicadas. Tais contratempos foram trabalhados e usados a favor dos alunos, com o intuito de fazer com que eles se vissem motivados a tentarem quantas vezes fossem necessárias para se chegarem à resolução correta da questão.

Ao final, verificou-se que aqueles alunos que realmente se propuseram a dar atenção às explicações e a fazer as tarefas aplicadas, conseguiram aprender o conteúdo que estava sendo trabalhado. Este fato tornou a oficina exitosa, afinal de contas o objetivo era fazer com que a maioria dos alunos, entendendo o sentido intuitivo dos cálculos apresentados, conseguindo compreender assim, o conteúdo demultiplicação entre matrizes.

Com isso, espera-se que os alunos, depois da oficina, se vejam motivados a aprenderem a matemática olhando para situações do dia a dia e que eles consigam se superar mesmo quando o conteúdo estudado não seja, perante o currículo escolar oficial, do nível ao qual se encontram. 
Antonio Ramon Firmo da Costa, Vaniele Sousa Alves e Nilton José Neves Cordeiro

Utilizando a conta de energia elétrica para o ensino do processo de multiplicação entre matrizes

Aos aplicadores, a experiência com os alunos do $1^{\mathrm{O}}$ ano do ensino médio serviu

como laboratório metodológico e didático. Metodológico por que a forma como o conteúdo foi repassado usou uma prática alternativa que busca mostrar aos alunos que a matemática está presente ao redor nas suas vidas. Didático por que houve momentos em que foi necessária apresentação de conteúdo, e essa explanação, de forma clara e compreensível, só foi possível graças à forma como foi feita as comparações e exemplos no momento da explicação.

\section{Referências}

ALMEIDA, C. S.Dificuldades de aprendizagem em Matemática e a percepção dos professores em relação a fatores associados ao insucesso nesta área.2006. $13 \mathrm{f}$. Monografia (Trabalho de Conclusão de Curso de Graduação em Matemática) Universidade Católica de Brasília - UCB, Brasília, 2006. Disponível em: <https://www.ucb.br/sites/100/103/TCC/12006/CinthiaSoaresdeAlmeida.pdf $>$ Acesso em: 20 set. 2016.

ÁVILA, T. P. As operações aritméticas e a aprendizagem de matrizes no ensino médio.2013. 37 f. Dissertação (Mestrado Profissional em Ensino de Ciências e Matemática) Universidade Cruzeiro do Sul - UNICSUL, São Paulo, 2013. Disponível em: <http://www.cruzeirodosul.edu.br/wp-content/uploads/2016/03/PE_TiagoPereira\%C3\%81vila-2013-v-publicada.pdf $>$. Acesso em: 20 fev. 2017.

BRASIL. Secretaria de Educação Básica. Ciências da Natureza, matemática e suas tecnologias: Orientações curriculares para o ensino médio, v. 2. Brasília: MEC, 2006.

CARVALHO, D. L. Metodologia do ensino da matemática. Cortez Editora, 1994.

CRUZ,R. Ensino de multiplicação de matrizes via transformações geométricas. 2012. 83f. Monografia (Graduação em Matemática) -Universidade Federal do Rio Grande do Sul - UFRGS, Porto Alegre, 2012. Disponível em:

<http://hdl.handle.net/10183/66864> Acesso em: 24 set. 2016.

PINTO, N. B. O erro como estratégia didática no ensino da matemática elementar. 1998. 320f. Tese (Doutorado em Matemática) - Universidade de São Paulo - USP, São Paulo, 1998. Disponível em: <http://www.teses.usp.br/teses/disponiveis/48/48133/tde12022015-151819/pt-br.php>. Acesso em: 24 set. 2016.

POLYA, G.A arte de resolver problemas. Editora Interciência, 1995.

SOUZA, P. A.; LOPES, A. M. A.; AZEVEDO, C. L. V. R.O estudo de produto de matrizes por meio de um objeto de aprendizagem. XI Encontro Nacional de Educação Matemática, 2013, Curitiba, Paraná. Anais do $11^{\circ}$ Encontro Nacional de Educação Matemática. Brasília: SBEM, 2013. p.1-8.Disponível em: $<$ http://sbem.web1471.kinghost.net/anais/XIENEM/pdf/1250_1364_ID.pdf >. Acesso em: 20 fev. 2017. 
Antonio Ramon Firmo da Costa, Vaniele Sousa Alves e Nilton José Neves Cordeiro

Utilizando a conta de energia elétrica para o ensino do processo de multiplicação entre matrizes

UTFPR. Disponível em: <http://www.utfpr.edu.br/patobranco/estrutura-

universitaria/assessorias/ascom/arquivos/sala\%20de\%20aula\%20novas\%20cadeiras $\% 2$

0e\%20carteiras.jpg >. Acesso em: 20 de set. 2016. 